\title{
Morphological Features and Molecular of Plasmodium inui in Macaca fascicularis from Bogor, West Java
}

\author{
Upik Kesumawati $^{1}$, Lis Rosmanah ${ }^{2 *}$, Susi Soviana ${ }^{1}$, Uus Saepuloh ${ }^{2}$, Huda \\ Shalahudin Darusman ${ }^{1,2}$
}

${ }^{1}$ Faculty of Veterinary Medicine, IPB University, Bogor, Indonesia

${ }^{2}$ Primate Research Center IPB University, Bogor, Indonesia

*Corresponding atuhor. E-mail: lisrosmanah@gmail.com

\begin{abstract}
Plasmodium is causative agent of malaria in human through the intermediary of female mosquitoes Anopheles spp. Two of the most important human malaria parasites, $P$. falciparum and $P$. vivax, are derived from the complete transmission event of non-human primate malaria species to human. Late data shows an increase number of malarial cases caused by $P$. malariae like Plasmodium parasite. The results of molecular analysis shows that such cases are not caused by $P$. malariae but by the primate malarial parasite, $P$. knowlesi. The $P$. cycomolgy is another plasmodium of non-human primate has also been through transmission events so that it is able to infect human. The aim of this study was to investigate the morphological features and molecular of Plasmodium inui in Macaca fascicularis from Bogor, West Java. Microscopic identification was carried out on thin blood smear prepared from blood samples collected from 274 M. fascicularis in two captivities in Bogor, West Java. Genomic DNA was extracted using QIAgen extraction kit and protocol, and used for SSU-RNA based PCR amplification and sequencing. The results showed the incidence of plasmodium in the Macaca fascicularis examined was $13.8 \%$ (38/274). Molecular analysis showed that the Plasmodium positive primates were infected by $P$. inui. The parasite was found in the trophozoite and schizon forms that are morphologically very similar to to that of Plasmodium malariae.
\end{abstract}

Keywords: Plasmodium, Plasmodium inui, Macaca fascicularis, microscopic identification

\section{INTRODUCTION}

Plasmodium is the causative agent of malaria in humans through the intermediary of female Anopheles spp. Plasmodium in humans such as Plasmodium vivax, $P$. malariae, $P$. ovale and $P$. falciparum. The two most important human malaria parasites, $P$. falciparum and $P$. vivax, are derived from the entire transmission event of non-human primate malaria species to humans. The latest data shows increased numbers of malaria cases caused by $P$. malariae like plasmodium. Result of molecular analysis shows that such cases are not caused by $P$. malariae but by nonhuman primate malaria parasites. $P$. knowlesi and $P$. cynomolgy are other plasmodiums of non-human primate have also been through transmission events so that they are able to infect humans.

Malaria parasites have a specific host, but recently the parasite infected Macaca fascicularis and $M$. nemestrina, $P$. knowlesi, significantly cause malaria in humans [1]. The P. knowlesi infection in human in
Thailand has been confirmed by using microscopic and PCR examinations [2]. The high infection of $P$. knowlesi in humans is likely to be infected with other plasmodium derived from non-human primate host [3] [4], meaning that today the parasites cause malaria in humans consist of 5 species namely $P$. falciparum, $P$. vivax, $P$. malariae, $P$ ovale and $P$. knowlesi.

Microscopic examination shows morphological resemblance of $P$. knowlesi to $P$. malariae and $P$. cynomolgi to $P$. vivax [5]. This may lead to errors in diagnosis, handling and treatment.

Identification is used two different methods which each have their own advantages and disadvantages. Thin blood swab is a cheap and fast conventional method but the drawbacks of this method cannot identify parasites if the level of parasitemia is low and requires a skill and experience in its identification. The second method of molecular identification, the advantages of this method can detect parasites with low levels of parasitemia and very high accuracy 
while the lack of workmanship is complicated and at considerable cost.

This research aims to identify microscopic and molecular Plasmodium from non-human primate, especially M. fascicularis.

\section{MATERIALS AND METHODS}

\subsection{Ethical Clearance}

Al methods used in this research have been aproved by the IPB Ethics Commission No. PRC-20EOO5.

\subsection{Ethical Clearance}

Blood samples were collected from $274 \mathrm{M}$. fascicularis in two captivities in Bogor, West Java. Blood, 2 ml per subject, was collected using EDTA as anticoagulant, and used for microscopic and molecular analyses.

\subsection{Microscopic Analysis}

A thin smear was prepared from $10 \mu \mathrm{l}$ of whole blood on an object glass, fixed with absolute methanol, and stained with Giemsa staining solution for 30 minutes. The presence of malaria parasite was microscopically observed using a magnification of 1000x.

\subsection{DNA Extraction}

The genomic DNA was extracted using QIAmp DNA Mini kit. Blood samples, $200 \mu \mathrm{l}$, was added with $200 \mu \mathrm{l}$ of AL lysis buffer and $20 \mu \mathrm{l}$ of proteinase-K. The mixture was incubated at $56{ }^{\circ} \mathrm{C}$ for 10 minutes, added with $200 \mu \mathrm{l}$ of $95 \%$ ethanol, and homogenized. All mixtures were transferred to a spin column in a collection tube and centrifuged at $6,780 \mathrm{xg}$ for 1 minute. Filtrate was discarded, the column was washed with AW1 and AW2 buffers. The column was transferred to a sterile $1.5 \mathrm{ml}$ microtube, added with $100 \mu \mathrm{l}$ of EB buffer, and incubated at room temperature for 2 minutes. The column was spun at $6,780 x g$ for 1 minute, and the filtrate (DNA extract) was stored at $-20{ }^{\circ} \mathrm{C}$.

\subsection{PCR Amplification}

The target gene for PCR amplification of Malaria Plasmodium was partial of small subunit ribosomal RNA (SSU rRNA) gene. One set primer used in this PCR was adapted from Cox-Singh and Sing [5] namely rRPLU1 and rRPLU5 that specifically ampligy a 1640-bp amplicon. The PCR amplification was carried out using thermalcycler machine (GeneAmp® PCR System 9700, Applied
Biosystem $^{\mathrm{TM}}$, Foster City, CA, USA). A total volume of $25 \mu \mathrm{L}$ reaction containing $10 \mathrm{pmol} / \mu \mathrm{l}$ of each primer (IDT, Singapore), $12.5 \mathrm{uL}$ of GoTaq ${ }^{\circledR}$ Green Master Mix (Promega, Madison, WI, USA), $2.5 \mu \mathrm{L}$ of $25-50 \mathrm{ng} / \mu \mathrm{L}$ of DNA templates was prepared. The condition of PCR process (40 cycles) was predenaturation at $94{ }^{\circ} \mathrm{C}$ for $3 \mathrm{~min}$, denaturation at $94{ }^{\circ} \mathrm{C}$ for $30 \mathrm{~s}$, annealing at $56^{\circ} \mathrm{C}$ for $30 \mathrm{~s}$, and extension at $72{ }^{\circ} \mathrm{C}$ for $30 \mathrm{~s}$, and the final extension at $72{ }^{\circ} \mathrm{C}$ for 5 min. The products were visualized using $1.8 \%$ agarose gel electrophoresis stained with SYBRsafe ${ }^{\mathrm{TM}}$ DNA Staining (Thermo Scientific, USA) and detected using Gel Doc 2000 (Bio-Rad, USA).

\subsection{Sequencing}

Determination of plasmodium species was carried out based on the nucleotide sequence of RNA (SSU rRNA) gene sequenced using the same primer set as the PCR amplification process. Analysis of sequencing results was conducted using Bioedit program and aligned to the data availabe at the Gen Bank (NCBI) using Blast Clustering program. The SSU rRNA based pylogenetic tree was constructed using Mega-6 software.

\section{RESULTS AND DISCUSSION}

The microscopic and molecular identifications of Plasmodium in the Macaca fascicularis from two captivities in Bogor of West Java had been done. Microscopic examination showed that plasmodium was positively identified in $13.87 \%$ (38/274) of $M$. fascicularis. The incidence was relatively small compared to the incidence reported in Malaysia, where $97.33 \%(73 / 75)$ of $M$. fascicularis were positively infected by the parasite [6]. Similarly, 93.54\% (29/31) of orangutans in rehabilitating facilities were infected by plasmodium [7]. This low incidence might be related to good sanitary conditions and food nutritional value as well as non malarial endemic status of study location. This distinguishes the results of this study from those of Foster et al. [6], who used samples from malarial endemic regions.

The morphology of Plasmodium can be microscopically observed in red blood cells as oval, round, or ring-shaped bluish black spots, depending upon the life cycle of parasites in animals at the time of blood collection (Figure 1). The cycle of non-human primate malaria in the blood starts with trophozoite with a ring shape that then enlarges and grows into an amoeba-like form.

The trophozoite continues to enlarge and change to schizont. After transforming into young gametocytes, macrogametocytes and microgametocytes, the parasite fill the entire volume of red blood cell. 
Using microscopic observation alone is very difficult to determine Plasmodium species that infect animals. Microscopic identification of Plasmodium species is time consuming and requires good skills and long experience due to morphological similarity of the early tropozoite stage of $P$. knowlesi to those of $P$. malariae and P. falciparum [8]. This method was, carried out in this study to determine the genus of the parasite.

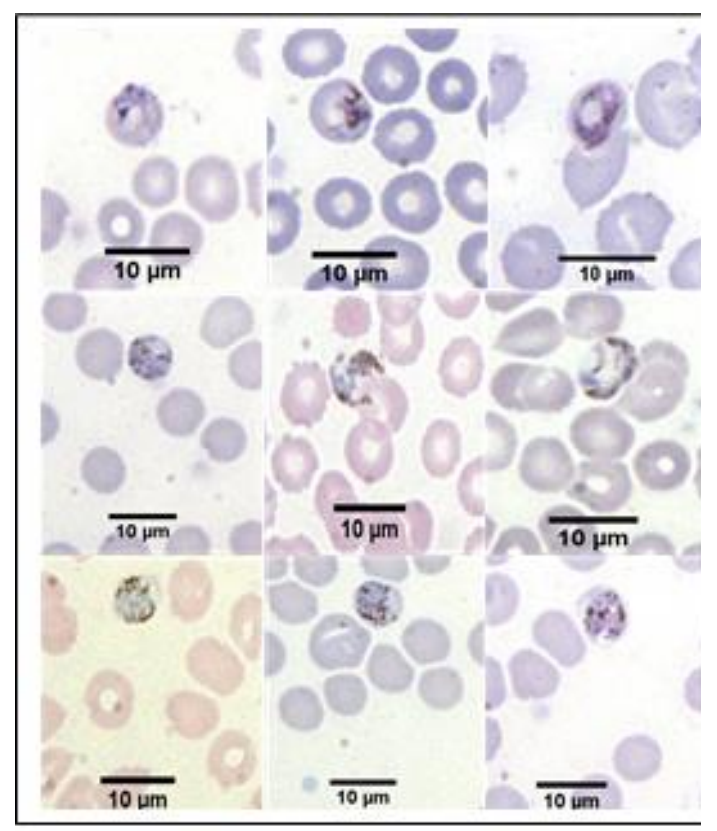

Figure 1 Tropozoite and schizon forms of Plasmodium inui (1000x magnification).

This study used molecular approach involving PCR amplification, sequencing and sequence analysis of SSU-rRNA gene to determine Plasmodium species infects $M$. fascicularis in Bogor. The nucleotide alignment using the NCBI Blast Program showed that all Plasmodium isolated from the macaca resembled to $P$. inui with a $99 \%$ of percent identity. The phylogenetic analysis confirmed the case as a single $P$. inui infection.

Biological characteristics of $P$. inui are similar to those of $P$. malariae [2]. Although $P$. inui infection in humans was only reported experimentally, this similarity gives the possibility of increasing the risk or zoonotic potential of the parasite. Therefore, it is important to further characterize Plasmodium species causing malaria in both human and non-human primates.

The results of this study provided important information about Plasmodium species that infects $M$. fascicularis, especially in West Java where the case was previously unreported. The discovery of $P$. inui in non-human primate could add more information about the distribution of Plasmodium in Indonesia.

\section{CONCLUSION}

This study obtained morphological forms of Plasmodium inui from the stage of ring form, trophozoite and schizont in M. fascicularis in Bogor, West Java. The molecular characterization confirmed infection the parasite in the primate.

\section{AUTHORS' CONTRIBUTIONS}

All authors equally contributed to the manuscript preparation, writing, and editing.

\section{ACKNOWLEDGMENTS}

This research was supported by the Directorate General of Higher Education, Ministry of Education and Culture of the Republic of Indonesia through Basic Research of Excellent Higher Education (PDUPT) 2020-2021.

\section{REFERENCES}

[1] B. Singh, A. Bobogare, J. Cox-Singh, G. Snounou, M.S. Abdullah, H.A. Rahman, A genus- and species-specific nested polymerase chain reaction malaria detection assay for epidemiologic studies. Am. J. Trop. Med. Hyg. 60(4) (1999) 687-692. DOI: 10.4269/ajtmh.1999.60.687

[2] B. Singh, K.S. Lee, A. Matusop, A. Radhakrishnan, S.S.G. Shamsul, J. Cox-Singh, A.W. Thomas, D. Conway, A large focus of naturally acquired Plasmodium knowlesi infections in human beings, The Lancet 363(9414) (2004) 1017-1024. DOI: 10.1016/S0140-6736(04)15836-4.

[3] W.E. Collins, J.W. Barnwell, Plasmodium knowlesi: finally being recognized. Editorial Commentary, J. Infect. Dis. 199(8) (2009) 1107 1108. DOI: $10.1086 / 597415$.

[4] M. Figtree, R. Lee, L. Bain, T. Kennedy, S. Mackertich, M. Urban, Q. Cheng, B.J. Hudson, Plasmodium knowlesi in human, Indonesian Borneo. Emerg. Infect. Dis. 16(4) (2010) 672 674. DOI: $10.3201 /$ eid1604.091624

[5] J. Cox-Singh, B. Singh, Knowlesi malaria: newly emergent and of public health importance? Trends Parasitol. 24(9) (2008) 406410. DOI: $\underline{10.1016 / j . p t .2008 .06 .001}$

[6] D. Foster, J. Cox-Singh, D.S.A. Mohamad, S. Krishna, P.P. Chin, B. Singh, Evaluation of three rapid diagnostic tests for the detection of human infections with Plasmodium knowlesi, Malar. J. 13, 60 (2014). DOI: https://doi.org/10.1186/14752875-13-60 
[7] T.H. Ta, $\underline{\text { S. Hisam, M. Lanza, A.I. Jiram, N-P. }}$ Ismail, J.M. Rubio, First case of a naturally acquired human infection with Plasmodium cynomolgy, Malar. J. 13, 68 (2014) DOI: $10.1186 / 1475-2875-13-68$

[8] I. Vythilingam, Y.M. Noorazian, T. Huat, A.I. Jiram, Y.M. Yusri, A.H. Azahari, I. Norparina, A. Noorrain, S.L. Hakim, Plasmodium knowlesi in humans, macaques and mosquitoes in Peninsular
Malaysia. Parasites and Vectors 1, 26 (2008). DOI: $10.1186 / 1756-3305-1-26$

[9] N.D. Wolfe, W.B. Karesh, A.M. Kilbourn, J. Cox-singh, E.J. Bosi, H.A. Rahman, A.T. Prosser, B. Singh, M. Andau, A. Spielman, The impact of ecological conditions on the prevalence of malaria among orangutan. Vector Bone and Zoonotic Disease, 2(2) (2002) 97-103. DOI: $\underline{10.1089 / 153036602321131896}$ 